\title{
Cuing mechanisms in auditory signal detection
}

\author{
RONALD HÜBNER and ERVIN R. HAFTER \\ University of California, Berkeley, California
}

\begin{abstract}
Detection of auditory signals under frequency uncertainty can be improved by presenting cues to the listeners. Since various cues have been found to differ in effectiveness, three conceivable mechanisms were considered which might account for these differences. Cuing might reduce the number and/or width of the employed auditory filters or listening bands. Also, cues could modulate the precision of frequency tuning of the filters. Psychometric functions were collected in a detection experiment with frequency uncertainty employing three kinds of cues: pure tones whose frequency was identical to that of the signal (iconic cues), complex tones with a missing fundamental equal to the signal (complex cues), and pure tones with a certain frequency relation to the signal (relative cues). Compared with a no-cue condition, all cue types improved detection performance. Fitting models to the data suggests that in the no-cue condition as well as the complex-cue condition, multiple bands were utilized, and that the iconic and relative cues induced single-band listening. There is no indication that accuracy of frequency tuning was responsible for cue-efficiency differences.
\end{abstract}

For the detection of sinusoidal auditory signals in noise, trial-by-trial variation of signal frequency results in lower performance than when it is fixed (e.g., Creelman, 1960; Green, 1961; Swets \& Sewall, 1961). However, this uncertainty effect can be compensated for by providing clearly audible cues at the beginning of each trial.

Various kinds of cues have been shown to be effective. These include what Schlauch and Hafter (1991) called "iconic cues," which match the signal in frequency, and cues that provide less direct information about the signal's frequency. The latter include multitonal cues for which only one component matches the signal (Gilliom \& Mills, 1976; Schlauch \& Hafter, 1991), visual cues in which one of two frequencies is cued with lights (Swets \& Sewall, 1961), and tonal complexes whose fundamental is related to the signal (Hafter, Schlauch, \& Tang, 1993).

Cues based on musical relations (Hafter et al., 1993) and on tonal patterns (Howard, O'Toole, Parasuraman, \& Bennett, 1984) or visual patterns (Howard, O'Toole, $\&$ Rice, 1986) related to the signal have also been applied successfully, and, for listeners with absolute pitch, so have visually presented musical notes (Hafter \& Schlauch, 1992).

Although all these cue types may serve to reduce frequency uncertainty, there are nevertheless appreciable differences in their efficiency (e.g., Hafter et al., 1993; Schlauch \& Hafter, 1991). Generally, it can be said that

\footnotetext{
This research was conducted while the first author was a visiting scholar at the Psychology Department of the University of California at Berkeley as holder of a NATO science fellowship. The authors would like to thank the unknown reviewers for valuable comments which helped us to improve on the former versions of this paper. Correspondence should be addressed to the first author, who is now at the Institut für Psychologie, Technische Universität Braunschweig, Spielmannstr. 19, D-38106 Braunschweig, Germany (e-mail: t.huebner@, tu-bs.de).
}

iconic cues are the most effective, usually eliminating the frequency-uncertainty effect completely.

How can the observed efficiency differences between different cue types be explained? Possible answers depend on the specific signal detection model under consideration. Usually it is assumed that under optimal conditions, detection of tonal signals in wideband noise is based on the output of auditory filters centered near the signal's frequencies. For instance, in a two-interval forcedchoice (2IFC) experiment, where a decision has to be made about which one of two temporal intervals contains the signal, it is assumed that listeners choose the interval containing the largest filter output. Although less agreement exists with respect to what the filter outputs represent (see, e.g., Jeffress, 1964), the energy detection model, which assumes that the filter outputs correspond to stimulus energy, is widely used to describe auditory detection (cf. Green \& Swets, 1966).

When the signal is a pure tone with a single fixed frequency, it is reasonable to assume the use of a single bandpass filter whose center frequency matches that of the signal. However, when there is frequency uncertainty, most theorists have proposed that the listener simultaneously monitors the outputs of multiple filters (Green, 1961; cf. Swets, 1984). To obtain a single decision variable for this case, one must devise a combination rule for the multiple outputs. One rule (Creelman, $1960)$ that is widely used says that the subject chooses the interval that has the maximum output of any of the monitored filters. The more filters, the more likely it will be that the maximum output will be due to noise alone, thus lowering performance. Another rule is to construct a weighted sum of the outputs, thereby increasing the effective noise, which also predicts a decrease in performance. Within these multiple-band conceptualizations, cuing-efficiency differences can be modeled according to their capacity to reduce the number of monitored bands. 
However, it has also been argued that there are "listening bands" whose widths are labile and that an effect of frequency uncertainty is to widen these listening bands (Hafter \& Kaplan, 1976). Since band widening increases the amount of effective noise, detection performance drops. In this case, it is reasonable to suppose that cuing reduces uncertainty by reducing the effective bandwidths. This hypothesis received support from recent results of Hafter et al. (1993). They employed a modified version of the so-called probe-signal method (Dai, Scharf, \& Buus, 1991; Greenberg \& Larkin, 1968; Schlauch \& Hafter, 1991) to measure the width of listening bands during detection with iconic and relative cues. The latter were tones with two thirds of the signal frequency. It turned out that the relative cues were less effective than the iconic cues and that this difference was attributable to bandwidth differences.

The models considered so far usually ignore possible effects of filter shape. However, auditory filters are modeled as having sloping skirts (Patterson \& Moore, 1986), which implies that performance is maximal for a chosen filter only when it is centered on the signal frequency, or, to put it another way, when its frequency tuning is optimal. Any shift would produce an attenuation of the signal and, consequently, a decrease in detection performance. This fact offers a third mechanism possibly related to cuing. The efficiency differences of various cue types could reflect the accuracy of frequency tuning that they permit.

Thus, at least three different mechanisms might account for cue effects and their differences. Fortunately, as has been shown by one of the present authors (Hübner, 1993), there is a method to discriminate empirically between the considered cuing mechanisms. Idealobserver analysis reveals that each mechanism affects psychometric functions in a specific way. Common to all mechanisms is that decreasing efficiency is reflected by a shift of the psychometric functions toward higher levels. However, there are differences concerning the slopes. Whereas inaccurate frequency tuning leads to flattened psychometric functions, increasing the width or number of the auditory filters causes just the oppositethe functions steepen.

Unfortunately, the predicted slope differences are rather small for usual experimental situations and so might not be detected. For instance, even in conditions with frequency uncertainty without cues, Green (1961) found slopes that were similar to those obtained with fixed frequencies. He drew this conclusion, however, by visually inspecting collections of functions from different situations, and since he observed only small frequencyuncertainty effects for most of his conditions, the question of whether a small slope difference could have been shown at least for the condition that produced the largest effect remained open. In contrast, Schlauch and Hafter (1991) showed appreciable changes in the slopes of psychometric functions as the amount of uncertainty increased.
In another related study, Buus, Schorer, Florentine, and Zwicker (1986) randomized one complex and three pure tones and compared the resulting psychometric functions with those obtained with fixed signals. Although they did not employ a consistent within-subjects design, they found a significant slope difference at least for one frequency.

The aim of the experiment reported here was to provide further evidence for the idea that the slopes are affected in a systematic manner by frequency uncertainty and to utilize that information for distinguishing between mechanisms possibly involved in cuing. Therefore, psychometric functions were collected in a signal detection experiment with frequency uncertainty for different cue types: iconic, complex, and relative cues. The complex cues were harmonic tones that had a missing fundamental equal to the signal. On the basis of past results (Hafter \& Schlauch, 1992), we expected the iconic cues to be the most, and the relative cues the least effective. The variation in the slopes of the psychometric functions with decreasing efficiency should provide information about the considered cuing mechanisms.

\section{METHOD}

The signals were pure tones of one of eight frequencies: 535 , $625,755,835,975,1025,1145$, and $1285 \mathrm{~Hz}$. In a random-frequency condition, all signals were randomly presented with equal probability. Three different kinds of cues were employed: iconic, complex, and relative. A control condition with no cues was also included. The complex cues consisted of the 2 nd, $3 \mathrm{rd}, 4 \mathrm{th}, 5 \mathrm{th}$, and 6 th harmonics of the respective signal frequencies. The relative cues were pure tones with two thirds the frequency of the respective signal.

An accelerated adaptive 2IFC procedure (Falmagne, 1985) was used to measure the fixed-frequency thresholds of the individual tones. The obtained thresholds correspond to $70.7 \%$ correct responses. The iconic and relative cues were presented at a level of $10 \mathrm{~dB}$ above this threshold. The complex cues were presented at a level such that their components were $10 \mathrm{~dB}$ above the threshold of the corresponding signals.

The stimuli were generated by a D/A-converter connected to an IBM-AT computer, which also controlled the presentation of the stimuli and registered the responses of the subjects. The stimuli, which had abrupt onsets and offsets, were mixed with continuously present white noise with a noise power density of $35 \mathrm{~dB}$ (SPL) and presented monaurally through a Stax SR5 headphone. The subjects were seated in a soundproof booth.

The cues had the same duration, $T$, of $0.2 \mathrm{sec}$ as the signals and were presented $0.5 \mathrm{sec}$ before the first interval. The two intervals, which were indicated by lights, were separated by $0.3 \mathrm{sec}$. Visual feedback was given after each response.

Three normally hearing subjects participated in the experiment, which was divided into thirteen 1 -h sessions. Each session consisted of eight blocks of 80 or 96 trials. To obtain estimates of the psychometric functions, levels of the individual tones were chosen to cover the range around $75 \%$ correct responses in a 2 IFC random-frequency condition. To average the performance over the different frequencies, sensation levels (SL) were used (i.e., the levels of the individual tones in decibels, relative to their fixedfrequency thresholds). With a stepsize of $2 \mathrm{~dB}$, this resulted in 5 to 6 points of the psychometric functions. Each point for each subject is the result of 400 trials. 


\section{RESULTS}

The psychometric functions of the 3 subjects and of their pooled data for the different conditions are depicted in Figure 1. Obviously, the iconic cues were most effective. However, the other cue types also improved detection performance in comparison with the no-cue condition.

Were the complex cues more effective than the relative cues? Their psychometric functions are rather close. However, for most levels, the data points of the complex cues are above the corresponding points of the relative cues. A Wilcoxon matched-pairs signed-ranks test with all 15 data pairs (subjects $\times$ levels) revealed a significant difference in the predicted direction $(T=30, N=$ $15, p<.05)$.

To get estimates of slopes and thresholds, logistic functions were fitted to the data by minimizing $\chi^{2}$ with a minimizing algorithm (Gegenfurtner, 1992). The results are displayed in the first three rows of Table 1 . Parameters were also estimated for the pooled data. They are given in the fourth row of the table. Generally, a good fit was obtained [e.g., for the pooled data, iconic: $\chi^{2}(3)=0.227$; complex, $\chi^{2}(2)=0.031$; relative, $\chi^{2}(2)=$ 0.148 ; no-cue, $\chi^{2}(2)=0.184 ; p>.90$ for all conditions].

Inspection of Table 1 shows that the efficiency order expected for the cue types is reflected by the order of each subject's estimated thresholds, except that there is one tie for Subject R.H. A different order, but also iden- tical for all subjects, with the exception again of one tie for Subject R.H., holds for the slopes.

To examine whether the slopes and threshold patterns hold across frequencies, the subjects' data were pooled, and logistic functions were fitted to the psychometric functions corresponding to the individual frequencies. No indication of a systematic relationship between slope and frequency could be found within each condition.

However, as a repeated measures analysis of variance (ANOVA) revealed, there were significant slope $[F(3,21)$ $=10.8, p<.001]$ and threshold $[F(3,21)=69.1, p<$ $.001]$ differences between the experimental conditions.

The Student-Newman-Keuls test was used for multiple pairwise comparisons. It turned out that the iconiccue condition produced significantly flatter psychometric functions (slopes: $M=0.0473$ ) than did the other conditions [comparison with the complex-cue condition, $M=0.0633, q(3,21)=6.10, p<.01$; with the relativecue condition, $M=0.0569, q(2,21)=3.67, p<.05$; with the no-cue condition, $M=0.0672, q(4,21)=7.54, p<.01]$.

While the slopes of the complex-cue condition were not significantly different from those of the relative-cue condition or from those of the no-cue condition, the relativecue condition produced significantly smaller slopes than did the no-cue condition $[q(3,21)=3.89, p<.05]$.

The iconic-cue condition also produced significantly lower thresholds $(M=0.61)$ than did all other conditions [comparison with the complex-cue condition, $M=$ $1.55, q(2,12)=5.50, p<.01$; with the relative-cue con-

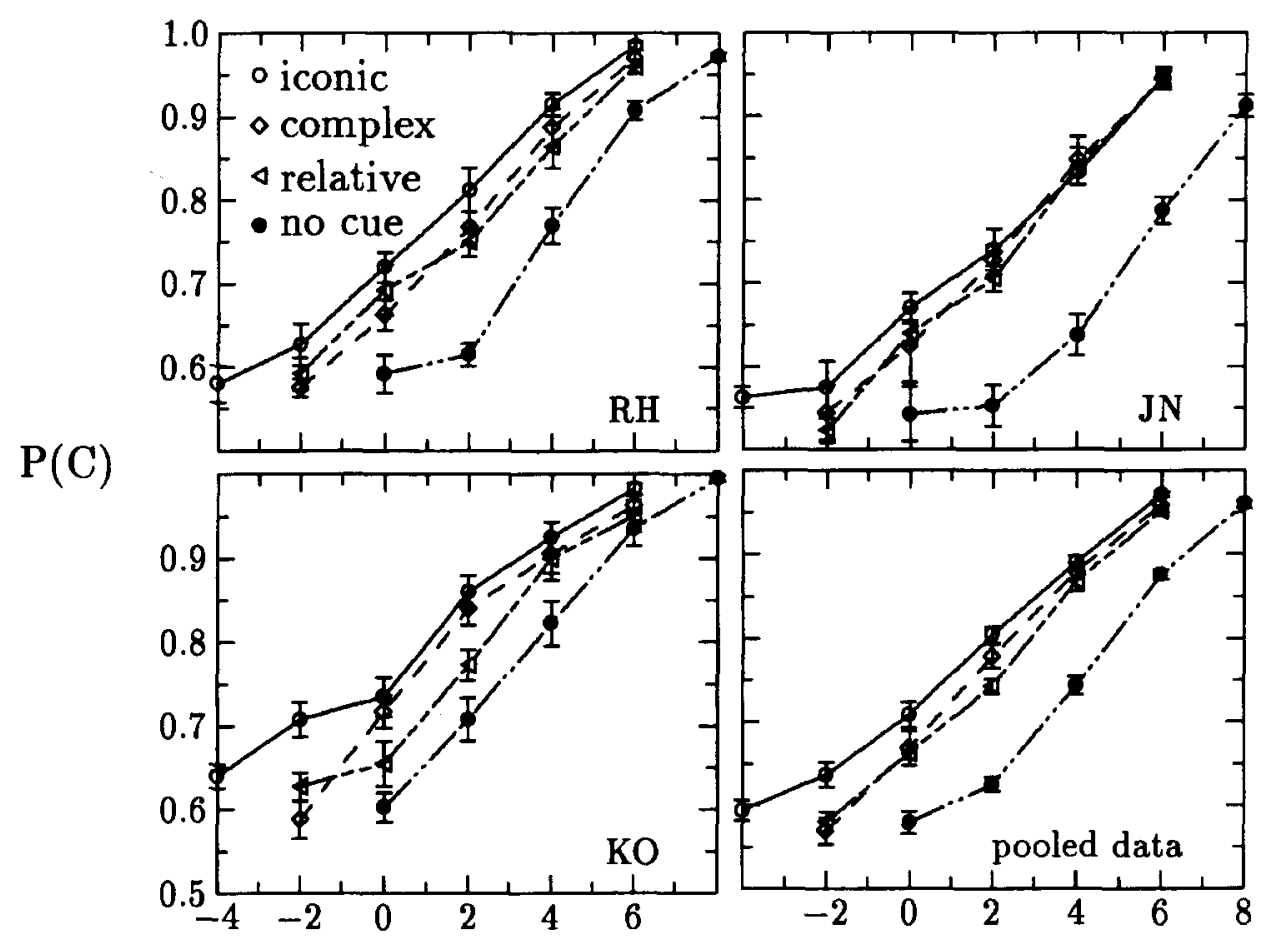

SL

Figure 1. Empirical psychometric functions of Subjects R.H., J.N., and K.O., and of their pooled data. The error bars indicate the standard error with respect to the experimental sessions. 
Table 1

Estimated Slopes and Thresholds of the Empirical Psychometric Functions for the 3 Subjects

\begin{tabular}{lccccccccc}
\hline & \multicolumn{4}{c}{ Slopes } & & \multicolumn{4}{c}{ Thresholds } \\
\cline { 2 - 4 } \cline { 8 - 10 } Subject & Iconic & Complex & Relative & No Cue & & Iconic & Complex & Relative & No Cue \\
\hline R.H. & 0.0534 & 0.0630 & 0.0534 & 0.0696 & & 0.45 & 1.51 & 1.51 & 3.62 \\
J.N. & 0.0502 & 0.0644 & 0.0621 & 0.0703 & & 1.80 & 2.36 & 2.61 & 5.54 \\
K.O. & 0.0419 & 0.0638 & 0.0521 & 0.0674 & & -0.77 & 0.65 & 1.28 & 2.65 \\
$M$ & 0.0484 & 0.0637 & 0.0559 & 0.0691 & & 0.66 & 1.51 & 1.80 & 3.94 \\
Models & 0.0468 & 0.0618 & 0.0557 & 0.0635 & & 0.62 & 1.56 & 1.87 & 3.98 \\
\hline
\end{tabular}

Note-All parameters were estimated by fitting logistic functions. The units of the slopes and thresholds are $P(\mathrm{C}) / \mathrm{SL}$ and $\mathrm{SL}$, respectively. In the fourth row, the average parameters are given, and the corresponding values predicted by the models are in the last row.

dition, $M=1.84, q(3,12)=7.20, p<.01$; with the nocue condition, $M=3.92, q(4,21)=19.7, p<.01]$. Thresholds of the complex-cue condition were not significantly lower than those of the relative-cue condition but were lower than those of the no-cue condition $[q(3,21)=$ $14.16, p>.01]$. The relative-cue condition also produced significantly lower thresholds than the no-cue condition $[q(2,21)=12.5, p<.01]$.

\section{DISCUSSION}

All cue types employed in the experiment improved detection behavior appreciably in comparison with the no-cue condition. Moreover, the psychometric functions corresponding to the different cue conditions showed significant slope differences. The iconic cues, which were most efficient, produced the flattest psychometric functions. Since all other conditions led to higher thresholds and to steeper psychometric functions, there is no indication that the decrease in cue efficiency can be attributed to poorer frequency tuning; this would have caused the functions to flatten (Hübner, 1993).

Therefore, we conclude that the observed performance differences were due to variations in the number and/or width of the utilized auditory filters. To examine the mechanisms in greater detail, different quantitative models were fitted to the psychometric functions for the individual frequencies (pooled across subjects) in each condition. For the sake of simplicity, only the values corresponding to the data points were predicted.

If we let $P(\mathrm{C})$ denote the probability of a correct response in a 2IFC task, then the energy-detection model states that

$$
P(\mathrm{C})=\Phi(z),
$$

where $\Phi$ denotes the cumulative normal distribution and $z$ is given by

$$
z=\frac{E\left(X_{s}\right)-E\left(X_{n}\right)}{\sqrt{\operatorname{var}\left(X_{s}\right)+\operatorname{var}\left(X_{n}\right)}} .
$$

In the case of a single band, the expected value of the decision variable $X_{n}$ in the noise interval is $2 W T$ and its variance $4 W T$, where $W$ denotes the bandwidth of the au- ditory filter in hertz, and $T$, the interval duration in seconds. In the interval containing signal plus noise, the expected value of $X_{s}$ is $2 W T+2 E_{s} / N_{0}$ and the variance $4 W T+8 E_{s} / N_{0}$, with $N_{0}$ denoting noise power density (cf. Green \& Swets, 1966).

\section{Iconic-Cue Condition: A Single-Band Model}

To obtain a starting point, a single-band energydetection model was fitted to each frequency's psychometric function of the iconic-cue data, employing time and noise parameters as in our experiment.

Since the energy-detection model is an ideal-observer model, it is more sensitive than our subjects. Although increasing the filter width, $W$, decreases sensitivity, this was not sufficient to fit the data well, because the resulting slope also varied with $W$. Therefore, the threshold was taken as a second free parameter for each psychometric function. The thresholds are needed for transforming the employed SL values to signal energy. Changing the threshold simply shifts the function without affecting its slope.

Varying both the threshold and the filter width, $W$, for each function by means of the algorithm mentioned above leads to good fits, although not all of the obtained values were reasonable (thresholds in decibels $[\mathrm{SPL}]=50.6,46.8,51.9,44.6,46.3,50.9,51.7$, 53.8 , and $W: 309,78,922,13,38,472,975,1573$, corresponding to frequency in ascending order). However, we were not interested in the absolute values within the cue conditions but in the relationships between the conditions.

Logistic functions were fitted to the obtained theoretical data to compare the resulting slopes and thresholds with the corresponding values estimated from the empirical data. A $t$ test for paired observations indicated no difference [slopes, $M_{\text {theo }}=0.0468, t(7)=0.74, p>.48$; thresholds, $\left.M_{\text {theo }}=0.61, t(7)=0.65, p>.53\right]$.

\section{Relative-Cue Condition: A Single-Band Model}

In the next step, a single-band model for the relativecue data was constructed by using the threshold parameters of the iconic-cue condition directly and by increasing the bandwidth parameters obtained in that condition linearly - that is, by employing two free parameters $a$ and $b$ : 


$$
W_{i}(\text { relative })=a W_{i}(\text { iconic })+b,
$$

for all frequencies $i=1, \ldots, 8$.

This model was fitted simultaneously to all eight psychometric functions. The obtained parameters are $a=$ 1.40 and $b=37.2\left[x^{2}(37)=14.4, p>.99\right]$. There were no significant differences to the empirical data [slopes, $M_{\text {theo }}=0.0508, t(7)=2.34, p>.05 ;$ thresholds, $M_{\text {theo }}=$ $1.92, t(7)=1.42, p>.19]$.

This result is in line with that of Hafter et al. (1993), who found that the bandwidth obtained with relative cues was 1.6 times greater than that obtained with iconic cues. Here, the width is 1.4 times larger plus a constant, which is surprisingly close.

\section{No-Cue Condition: A Multiple-Band Model}

Since the relative cues provide no energy at the signal frequency, it is reasonable to assume that similar threshold and width parameters hold for the no-cue condition, but that under this condition multiple filters are utilized. If multiple filters are utilized, a combination rule for the multiple outputs must be chosen (cf. Hübner, 1993). Here, we chose the weighted sum of the filter outputs as a decision variable:

$$
X^{*}=\sum_{i=1}^{8} g_{i} X_{i} .
$$

It was assumed that in the signal interval, the filters that do not correspond to the signal frequency behave as they do in the noise interval. Therefore, the expected value for the noise interval is

$$
E\left(X_{n}^{*}\right)=2 T \sum_{i=1}^{8} g_{i} W_{i}
$$

and the expected value for the signal-plus-noise interval is

$$
E\left(X_{n}^{*}\right)=2 T \sum_{i=1}^{8} g_{i} W_{i}+2 g_{s} E_{s} / N_{0},
$$

where $g_{s}$ denotes the respective weight of the signal channel.

It was further assumed that the auditory filters do not overlap and, consequently, do not produce correlated outputs. In this case, the outputs can be treated as independent random variables with the variance

$$
\operatorname{var}\left(X_{n}^{*}\right)=4 T \sum_{i=1}^{8} g_{i}^{2} W_{i}
$$

for noise, and

$$
\operatorname{var}\left(X_{n}^{*}\right)=4 T \sum_{i=1}^{8} g_{i}^{2} W_{i}+8 g_{s}^{2} E_{s} / N_{0}
$$

for signal plus noise. The resulting $z$ value for this model is

$$
z=\frac{g_{s} E_{s} / N_{0}}{\sqrt{2 T \sum_{i=1}^{8} g_{i}^{2} W_{i}+2 g_{s}^{2} E_{s} / N_{0}}} .
$$

While using the thresholds and width parameters based on fits to the relative-cue data, the weights $g_{i}$ for each filter were considered as free parameters. However, the data fit with this model was disappointing $\left[\chi^{2}(31)=74.3, p<\right.$ $.001]$. The increase in slope for the psychometric functions was underestimated (slopes, $M_{\text {theo }}=0.0426$ ), and the decrease in performance, overestimated (thresholds, $M_{\text {theo }}=6.22$ ). The latter could reflect overlapping auditory filters that led to overestimated variances. To keep the model relatively simple, merely two additional free parameters $a^{*}$ and $b^{*}$ were introduced for linearly modifying the variance part produced by the filter widths.

But how to increase the slopes? An ideal observer would choose weights $h_{i}, i=1, \ldots, 8$, which may vary with signal level (Green \& Swets, 1966):

$$
h_{i}=\frac{E\left(X_{i}^{s}\right)-E\left(X_{i}^{n}\right)}{\operatorname{var}\left(X_{i}^{n}\right)} .
$$

Fortunately, the modified model with decision variable

$$
X^{*}=\sum_{i=1}^{8} g_{i} h_{i} X_{i}
$$

and $z$ value

$$
z=\frac{h_{s} g_{s} E_{s} / N_{0}}{\sqrt{a^{*} 2 T \sum_{i=1}^{8} h_{i}^{2} g_{i}^{2} W_{i}+b^{*}+2 h_{s}^{2} g_{s}^{2} E_{s} / N_{0}}}
$$

turned out to fit the data quite well $\left[\chi^{2}(29)=3.38, p>\right.$ .995] and produced no significant differences [slopes, $M_{\text {theo }}=0.0633, t(7)=1.41, p>.20$; thresholds, $M_{\text {theo }}=$ $3.96, t(7)=1.42, p>.19]$.

The parameter values for modifying the variance are $a^{*}=0.694$, and $b^{*}=-2.42$. The obtained parameters for the weights are $0.073,0.132,0.142,0.180,0.124$, $0.110,0.140,0.097$. With one exception, the weights increase toward the central frequencies, which might represent the distribution of attentional resources across the different outputs.

\section{Complex-Cue Condition: A Multiple-Band Model}

Finally, we tried fitting a model to the complex-cue data. Although their slopes and thresholds were not significantly different from those for the relative-cue condition, the data could not be fitted in the same way-that is, by linearly increasing the filter width parameters of the model of the iconic-cue condition. The slopes were significantly underestimated [slopes, $M_{\text {theo }}=0.0480$, $t(7)=5.20, p<.01]$. (Remember that there are differences between the two cue conditions, as has been shown above with the raw data across subjects.) Therefore, a multiple-band model was applied. That complex cues induce multiple-band listening has already been suggested by Schlauch and Hafter (1991).

Since the complex cues consisted of five components and did not include the signal frequency, we assumed a 
model with six bands, each possessing the width and threshold parameters of the respective signal band, which were taken from the model of the relative-cue condition. Ideal weights $h_{i}$ (see Equation 10) were assumed for the signal band and a linearly related weight $h_{i}^{\text {comp }}$ for all cue-component bands with free parameters $a$ and $b$ :

$$
h_{i}^{\text {comp }}=a h_{i}+b .
$$

As for the no-cue data, two additional parameters $a^{*}$ and $b^{*}$ were used to modify the variance attributable to the filter widths. The $z$ value for each $i=1, \ldots, 8$ in this case is

$$
z=\frac{h_{i} E_{s} / N_{0}}{\sqrt{a^{*}\left[10\left(h_{i}^{\text {comp }}\right)^{2} W_{i} T+2 h_{i}^{2} W_{i} T\right]+b^{*}+2 h_{i}^{2} E_{s} / N_{0}}} .
$$

With these four free parameters, the eight psychometric functions fitted well $\left[\chi^{2}(35)=10.9, p>.995\right]$ and produced no significant differences [slopes, $M_{\text {theo }}=$ $0.0563, t(7)=2.29, p>.05$; thresholds, $M_{\text {theo }}=1.54$, $t(7)=0.05, p>.96]$.

The obtained parameters are $a=0.471, b=0.0048$, and $a^{*}=0.377, b^{*}=-0.784$.

\section{Conclusions}

In Figure 2, the pooled data and the theoretic psychometric functions averaged across frequencies are shown. As can be seen, the fits are quite good. Estimating the slope and threshold parameters in the same way as for the empirical data produces the values given in the last row of Table 1, which are rather close to their empirical counterparts.

To sum up, our analysis shows that frequency uncertainty (with no cues) leads to multiple-band listening. Our results are compatible with the idea that a weighted

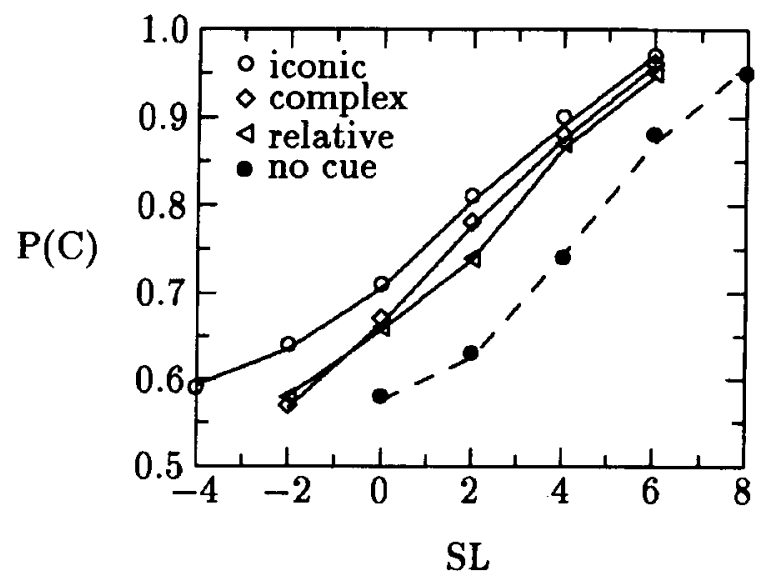

Figure 2. The pooled data with curves predicted by the models. sum of the outputs of the individual bands, which correspond to the possible frequencies, is utilized as decision variable. Also, complex cues lead to multiple-band listening. Presentation of iconic or relative cues induces utilization of single auditory filters with iconic cues causing smaller bandwidths.

\section{REFERENCES}

Buus, S., Schorer, E., Florentine, M., \& Zwicker, E. (1986). Decision rules in detection of simple and complex tones. Journal of the Acoustical Society of America, 80, 1646-1657.

Creelman, C. D. (1960). Detection of signals of uncertain frequency. Journal of the Acoustical Society of America, 32, 805-810.

DaI, H., Scharf, B., \& BuUs, S. (1991). Effective attenuation of signals in noise under focused attention. Journal of the Acoustical Society of America, 89, 2837-2842.

Falmagne, J.-C. (1985). Elements of psychophysical theory. New York: Oxford University Press.

GegenfurtneR, K. R. (1992). PRAXIS: Brent's algorithm for function minimization. Behavior Research Methods, Instruments, \& Computers, 24, 560-564.

GILliom, J. D., \& MiLLs, W. M. (1976). Information extraction from contralateral cues in the detection of signals of uncertain frequency. Journal of the Acoustical Society of America, 59, 1428-1433.

GREEN, D. M. (1961). Detection of auditory sinosoids of uncertain frequency. Journal of the Acoustical Society of America, 33, 897-903.

Green, D. M., \& SwETS, J. A. (1966). Signal detection theory and psychophysics. New York: Wiley.

GreEnBERG, G. Z., \& LaRkIn, W. D. (1968). Frequency-response characteristic of auditory observers detecting signals of a single frequency in noise: The probe-signal method. Journal of the Acoustical Society of America, 44, 1513-1523.

HAFTER, E. R., \& KAPLAN, R. (1976). Report on attention and flying. Unpublished manuscript prepared for NASA Ames Research Center.

Hafter, E. R., \& Schlauch, R. S. (1992). Cognitive factors and selection of auditory listening bands. In A. L. Dancer, D. Henderson, R. J. Salvi, \& R. P. Hamernik (Eds.), Noise-induced hearing loss (pp. 303-310). St. Louis: Mosby.

HafTer, E. R., Schlauch, R. S., \& TANG, J. (1993). Attending to auditory filters that were not stimulated directly. Journal of the Acoustical Society of America, 94, 743-747.

howard, J. H., O'Toole, A. J., Parasuraman, R., \& Bennett, K. B. (1984). Pattern-directed attention in uncertain-frequency detection. Perception \& Psychophysics, 35, 256-264.

Howard, J. H., O'ToOLE, A. J., \& Rice, S. E. (1986). The role of frequency versus informational cues in uncertain frequency detection. Journal of the Acoustical Society of America, 79, 788-791.

HÜBNER, R. (1993). On possible models of attention in signal detection. Journal of Mathematical Psychology, 37, 266-281.

JEFFrESs, L. A. (1964). Stimulus-oriented approach to detection. Journal of the Acoustical Society of America, 63, 766-774.

Patterson, R. D., \& MoOre, B. C. J. (1986). Auditory filters and exitation patterns as representations of frequency resolution. In B. Moore (Ed.), Frequency selectivity in hearing (pp. 123-177). London: Academic Press.

SChlauch, R. S., \& HafTER, E. R. (1991). Listening bandwidths and frequency uncertainty in pure-tone signal detection. Journal of the Acoustical Society of America, 90, 1332-1339.

SWETS, J. A. (1984). Mathematical models of attention. In R. Parasuraman \& D. Davis (Eds.), Varieties of attention (pp. 183-242). New York: Academic Press.

Swets, J. A., \& Sewall, S. T. (1961). Stimulus versus response uncertainty in recognition. Journal of the Acoustical Society of America, 33, 1586-1592.

(Manuscript received February 12, 1993; revision accepted for publication August 25, 1994.) 